\title{
Identification of External Force Acting on a Machine or a Structure in the Case of Unknown Force Location*
}

\author{
Shozo KAWAMURA**, Shintaro HORI** and Hirofumi MINAMOTO** \\ **Toyohashi University of Technology, Department of Mechanical Engineering \\ 1-1 Tempaku, Toyohashi, Aichi, 441-8580, Japan \\ E-mail: kawamura@mech.tut.ac.jp
}

\begin{abstract}
The identification of external force acting on a machine or a structure is important for the diagnosis. One of the force identification methods is based on the frequency response function. The method is very useful although it requires the knowledge of location of force acting. In this study, an identification method is proposed for the case of unknown excitation points. The method is firstly the external forces, whose amount is same as the one of sensors, are assumed at every candidate location, and the amplitudes of forces are identified. Then the exact location of external force is determined by the classification of all identified results. The method is numerically checked and it was shown that the method was useful using the data with noise. Moreover the experimental results showed that the method was feasible when the location and amplitude are separately identified.
\end{abstract}

Key words: Dynamics, Inverse Problem, Force Identification, Frequency Response Function, Singular Value Decomposition, Classification

\section{Introduction}

An external force acts on a machine during operation, or a dynamic force is generated by its operation. In the direct analysis procedure, the dynamic responses are calculated by setting the boundary condition, the initial condition and the shape of the analytical domain, but in some actual cases, the external force cannot be measured directly. Thus, to design and diagnose a machine or a structure, it is necessary to identify the external force acting on it. The identification is established as an inverse problem, and many studies of this problem have been performed in a variety of research fields ${ }^{(1),(2)}$.

To identify the external force, the frequency response function (FRF) is often used ${ }^{(3),(4)}$, but this requires knowledge of the location of the acting force, so it is difficult to apply the method if the location of the acting force is unknown. Numerical instability may occur according to the locations of the acting force and the measurement positions.

To overcome the problems of an unknown force location, a force identification method based on the equation of motion was proposed ${ }^{(5)}$, in which the dynamic response for all degrees of freedom was estimated by using the modal analysis method from a set of measurement data, and the external force was identified by substituting the response into the equation of motion. Although the exact response and the estimated response were not identical, this method is effective for systems with concentrated mass, for example, a rotor system, because the unbalance force can be identified at the rotor position. However, when the method is applied to uniform systems, for example, a beam system, the estimation error of the response has a large influence on the identified results, i.e., the concentrated force cannot be identified $^{(6)}$. Hori et al. proposed a selection method of locations of the external

${ }^{*}$ Received 17 July, 2009 (No. T1-07-0887) Japanese Original : Trans. Jpn. Soc. Mech. Eng., Vol.74, No.741, C (2008), pp.1122-1128 (Received 5 Oct., 2007) [DOI: 10.1299/jee.4.422] 
forces according the number of sensors ${ }^{(7)}$.

We propose a new method using the FRF to identify the magnitude and location of a force. In this method, by setting the number of external forces to equal the number of sensors, the external force is identified for all combinations of force location. Then, assuming the number of locations of external force, the pattern of identified results is checked so as to satisfy the condition according to the assumed number of locations of external force.

As a numerical example, the validity of the proposed method is checked by using the date without any measurement error and the applicability is checked by using the date with an error. We then checked the practicality experimentally.

\section{Outline of force identification using the FRF}

For simplicity of explanation, we ignore the damping here. The equation of motion of a machine or a structure is obtained by using Finite Element Method(FEM) as follows:

$$
[M]\{\ddot{x}\}+[K]\{x\}=\{f\},
$$

where $[M]$ and $[K]$ are the mass and stiffness matrices, respectively, and $\{x\}$ and $\{f\}$ are the response and external force vectors, respectively.

In this system, when the harmonic excitation

$$
\{f\}=\{F\} \exp (j \omega t)
$$

acts on the structure, the response can be assumed as follows:

$$
\{x\}=\{X\} \exp (j \omega t) .
$$

Substituting Eqs. (2) and (3) into Eq.(1), the equation of motion is obtained as follows:

$$
\left([K]-\omega^{2}[M]\right)\{X\}=\{F\} \text {. }
$$

Using this equation, the FRF matrix $[H(\omega)]$ can be obtained as follows:

$$
\{X\}=[H(\omega)]\{F\} .
$$

Here the response $\left\{X_{p}\right\}$ can be measured with $p$ sensors as follows:

$$
\left\{X_{p}\right\}=[C]\{X\},
$$

where matrix $[C]$ is the $(p \times n)$ matrix that expresses the locations of sensors. And when the external force acts at $q$ points on the structure whose locations are known, the vector $\left\{F_{q}\right\}$ is determined from $\{F\}$ by extraction of the location of the acting force. The relationship between the response $\left\{X_{p}\right\}$ and the external force $\left\{F_{q}\right\}$ can be written as follows:

$$
\left.\left\{X_{p}\right\}=\left\lfloor H_{p q}(\omega)\right\rfloor F_{q}\right\} .
$$

The result depends on the number of $p$ and $q$. This method can be applied when the location of the acting force is known in advance.

\section{Force identification in the case of unknown location of an acting force}

The external force can be identified using the procedure described in Section 2 when the location of the acting force is known, but the location of an acting force isn't always known. We propose an identification method using the FRF that applies in the case of unknown location of an acting force. The basic idea is that the force identification is carried out for all combinations of force location and the location is identified, and then the magnitude of the external force is determined considering the pattern of identified results.

When the responses are measured at $p$ points, the external force on up to $p$ points 
can be identified by using the least-squares method or the simultaneous equation. If the external forces act on $p$ points in the $n$ degrees of freedom system, then the number of combinations of force location is ${ }_{n} C_{p}$, and the correct result is only one. Here we can't find the correct result, so that the location and magnitude of the acting force of $(p-1)$ points are identified when the responses are measured at $p$ points. This means that we use the information that the magnitude of external force is zero at one point.

To explain the proposed method, the case of $p$ measurement points and $p$ acting forces is considered. In this case, Eq. (7) is written as follows:

$$
\left\{X_{p}\right\}=\left[H_{p p}(\omega)\right]\left\{F_{p}\right\} \text {. }
$$

First, the force identification is carried out for ${ }_{n} C_{p}$ combinations of force location. The FRF matrix in Eq. (8) is often in the ill-condition due to the measurement points and assumed locations of the acting force. In this case the significant small singular values are truncated. The procedure is as follows. The FRF matrix $\left\lfloor H_{p p}(\omega)\right\rfloor$ is expressed by using the singular value decompositions as follows:

$$
\left[H_{p p}(\omega)\right]=[U][B][V]^{T},
$$

where $[B]$ is the diagonal matrix composed of the singular values. Using Eqs. (8) and (9), the external force $\left\{F_{p}\right\}$ can be obtained as follows:

$$
\left\{F_{p}\right\}=[V][\bar{B}]^{-1}[U]^{T}\left\{X_{p}\right\},
$$

where $[\bar{B}]^{-1}$ is the diagonal matrix composed of the reciprocals of the singular values in which the significant small singular values are truncated.

Next, the results of ${ }_{n} C_{p}$ combinations are checked.

At first, the case of one external force is supposed. When the external force acts on the $l$-th component, the $l$-th component of the external force vector has a certain value and the other $(p-1)$ components show zero. So by checking the ${ }_{n} C_{p}$ results, and showing the same value at the $l$-th component and zero at other components, we can recognize that the external force only acts on the $l$-th component. And there are no patterns that satisfy the condition, which indicates that the external forces act on more than two points.

In the actual calculation, the truncation of small singular values and measurement error affect the identification results, and thus the numerical criteria for estimating the same value or zero are important. This is discussed in the numerical example.

Next, the case of two external forces is supposed. When the external forces act on the $l_{1}$-th and $l_{2}$-th components, the $l_{1}$-th and $l_{2}$-th components of the external force vector have certain values and the other $(p-2)$ components show zero. By checking the ${ }_{n} C_{p}$ results, and showing the same value at the $l_{1}$-th and $l_{2}$-th components and zero at the other components, we recognize that the external forces act on the $l_{1}$-th and $l_{2}$-the components. There are no patterns that satisfy the condition, which indicates that the external forces act on more than three points.

The proposed method is summarized as follows. First, by setting the number of external forces equal to the number of sensors, the external force is identified for all combinations of force location. Then, assuming the number of locations of external force, we check the pattern of identified results to satisfy the condition according to the assumed number of external forces. By using the proposed method, we can identify the external forces that act at (number of measurement points -1) points.

\section{Numerical Examples}

\subsection{Model for numerical example}

The mathematical model that expresses the experimental setup is shown in Fig. 1. The beam is divided into 20 elements, and the equation of motion is constructed by using FEM. 
The node number of the left end is Node 1, and the center of the beam is Node 11 . The beam is supported by two springs at Node 2 and Node 20, and the stiffness is added into the stiffness matrix of the beam.

The specifications are as follows:

Length: $1.0 \mathrm{~m}$, Moment of inertia: $1.63 \times 10^{-9} \mathrm{~m}^{4}$,

Young's modulus: $206 \mathrm{GPa}$, Density $7.65 \times 10^{3} \mathrm{~kg} / \mathrm{m}^{3}$,

Spring constant at Node 2: $27.4 \mathrm{kN} / \mathrm{m}$, Spring constant at Node 20: $26.4 \mathrm{kN} / \mathrm{m}$

The spring constants were actually measured. The damping was ignored because it was very slight by actual measurement.

We used four sensors $(p=4)$ for the experiment, and we measured the translational displacements at Nodes 4, 8, 14 and 18.

Some of the natural frequencies are shown in Table 1 . The compliance FRF $H_{4,11}$ and $H_{8,11}$ are shown in Fig. 2, which are the magnitude of displacement at Nodes 4 and 11 when a unit external force acts at Node 11. Some examples of measurement data are shown in Table 2 in the case of one, two and three excitations.

\subsection{Validity of the proposed method}

To check the validity of the proposed method, we identified the external force using the data without any measurement error. There are 42 degrees of freedom in the mathematical model, and we assumed that the external force acted only in the translational direction that has 21 degrees of freedom.

In the first example, we assumed that the external force whose frequency was $5.0 \mathrm{~Hz}$ and magnitude was $10.0 \mathrm{~N}$ acted at Node 11. The displacement of the beam is shown in Fig. 3, and the symbol $(\bigcirc)$ denotes the measurement data in the figure.

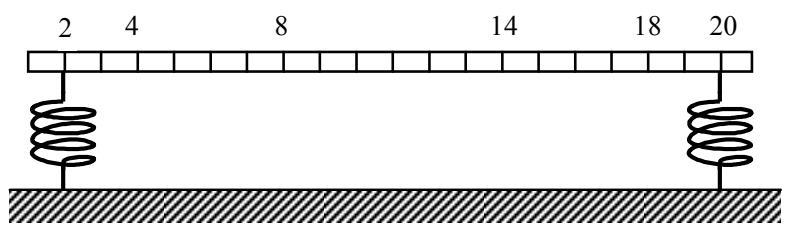

Fig.1 Model for numerical analysis

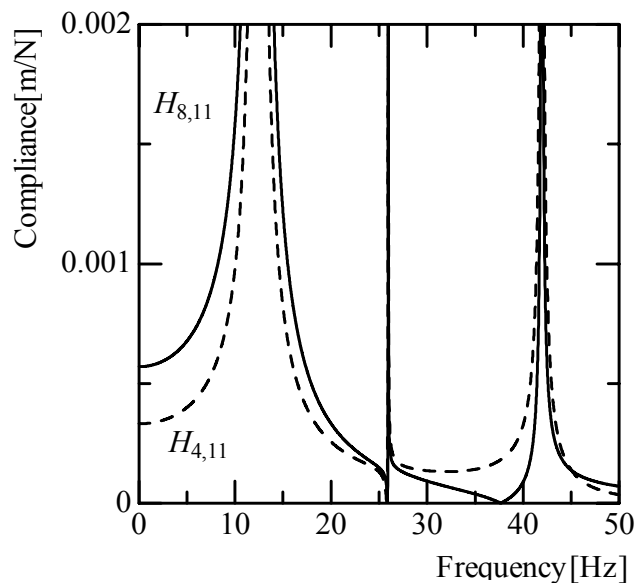

Table 1 Natural frequencies

\begin{tabular}{|c|c|}
\hline & Natural frequency $[\mathrm{Hz}]$ \\
\hline$f_{1}$ & 12.45 \\
\hline$f_{2}$ & 25.96 \\
\hline$f_{3}$ & 41.95 \\
\hline$f_{4}$ & 86.79 \\
\hline$f_{5}$ & 165.4 \\
\hline
\end{tabular}

Fig.2 Resonance curve

Table 2 Displacements at sensor locations

\begin{tabular}{|c|c|c|c|c|}
\hline & $x_{4}(\mathrm{~m})$ & $x_{8}(\mathrm{~m})$ & $x_{14}(\mathrm{~m})$ & $x_{18}(\mathrm{~m})$ \\
\hline One excitation & $3.9996 \times 10^{-4}$ & $6.7736 \times 10^{-4}$ & $6.8033 \times 10^{-4}$ & $4.0687 \times 10^{-4}$ \\
\hline Two excitations & $8.1029 \times 10^{-4}$ & $1.3722 \times 10^{-3}$ & $1.4217 \times 10^{-3}$ & $8.9285 \times 10^{-4}$ \\
\hline Three excitations & $1.2344 \times 10^{-3}$ & $2.0839 \times 10^{-3}$ & $2.1311 \times 10^{-3}$ & $2.3290 \times 10^{-3}$ \\
\hline
\end{tabular}




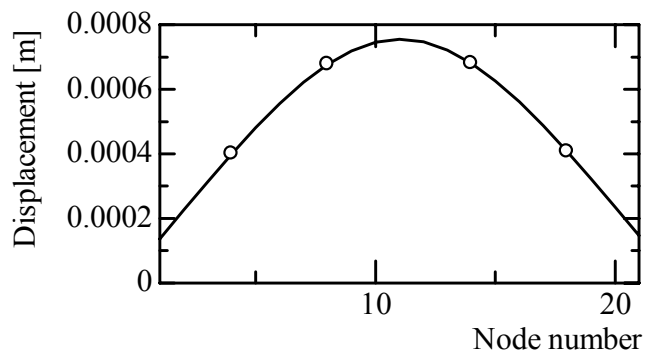

Fig. 3 Displacement at $5 \mathrm{~Hz}$

The external forces at four points are identified by using the measured data and Eq. (10). There are ${ }_{21} C_{4}(=5985)$ combinations in this example. The criteria of truncation of small singular values in Eq. (10) is set as $1.0 \times 10^{-8}$, which was determined as a threshold value where the singular value changes a lot. As a reference, the average value of the condition number is $7.4 \times 10^{13}$ when all singular values are adopted, while it is $1.2 \times 10^{3}$ when the small singular values are truncated. An example of identification results when the assumed four points of acting force include the correct location of the acting force is as follows:

$$
F_{5}=-4.0 \times 10^{-5}, \quad F_{11}=10.00, \quad F_{14}=-4.5 \times 10^{-5}, \quad F_{19}=-1.4 \times 10^{-5},
$$

while an example of identification results when they don't include the correct location is as follows:

$$
F_{5}=-4.35, F_{13}=8.96, F_{14}=6.43, F_{19}=-1.22 \text {. }
$$

Even if there is no error in the measured data, a very slight value remains at the location without external force because of the truncation of small singular values. This indicates the importance of the criteria for the estimation of zero. In this study, the value of $3 \%$ of the maximum magnitude of the identified external force is set as a threshold value to estimate zero by trial and error.

The estimation criteria regarding whether the two values agree are also important. In this paper, when the difference of the identification results is within $3 \%$ of the reference value determined first, the two results are estimated as being the same.

As an example, when the external force acts at one point, the ${ }_{20} C_{3}(=1140)$ combinations must follow the same patterns, but this result is not always performed because of the truncation of small singular values. In this study, the final identification result is determined when $90 \%$ of the ideal combinations are obtained in the identification results. In this case, the final result was determined when more than $1026(=1140 \times 0.9)$ combinations were obtained.

When one external force acts on the beam, the identification results can be classified. There were 1129 combinations when the magnitude of external force was almost $10.0 \mathrm{~N}$ at Node 11, and the magnitude was almost zero at three other locations. We thus concluded that one external force of $10.00 \mathrm{~N}$, which was an average value of 1129 results, acted at Node 11.

Next, two external forces of $5.0 \mathrm{~Hz}$ simultaneously acted on the beam whose properties are $10.0 \mathrm{~N}$ at Node 9 and $12.0 \mathrm{~N}$ at Node 14, respectively.

The external forces are identified by the method explained in Section.3. An example of identification results when the assumed four points of acting force include the correct location of acting force is as follows:

$$
F_{6}=1.0 \times 10^{-4}, F_{9}=10.00, F_{14}=12.00, F_{15}=-1.72 \times 10^{-4},
$$

while an example of identification results when they don't include the correct location is as follows:

$$
F_{6}=1.36, \quad F_{10}=8.49, \quad F_{12}=5.79, \quad F_{15}=6.37 \text {. }
$$

We first assumed that one external force acted on the beam. When the assumption was true, the combinations of ${ }_{20} C_{3}(=1140)$ had to agree well, but the condition wasn't satisfied, which indicated that the external forces acted at more than two locations. The 
results where the magnitudes of external force were the same at two locations and the magnitudes were zero at two other locations were checked, and the 171 results equal to ${ }_{19} C_{2}$ were found. We thus concluded that one external force of $10.0 \mathrm{~N}$ at Node 9 and one of $12.0 \mathrm{~N}$ at Node 14 acted on the beam.

Finally, three external forces of $5.0 \mathrm{~Hz}$ acted on the beam whose properties were $11.0 \mathrm{~N}$ at Node 9, 12.0 $\mathrm{N}$ at Node 11 and $10.0 \mathrm{~N}$ at Node 15. The external forces were identified by the method explained in Section 3. As the conditions indicating one and two excitations were not satisfied, we concluded that the external forces acted at more than three locations. The results where the magnitudes of external force were the same at three locations and where the magnitude was zero at one other location were checked, and 18 results were found, which was more than ${ }_{18} C_{1} \times 0.9$. Thus we concluded that one external force of 11.0 $\mathrm{N}$ at Node 9, one of $12.0 \mathrm{~N}$ at Node 11 and one of $10.0 \mathrm{~N}$ at Node 15 acted on the beam.

The results demonstrated the validity of the proposed method, because the exact identification result could be obtained by using the data without any measurement error.

\subsection{The applicability of the proposed method}

We tested the applicability of the proposed method using data with a measurement error. We were aware that there were some differences between the actual structure and its mathematical model, but in this study we felt that the sufficient mathematical model could be obtained at a certain frequency of force identification.

The approximate number to two, three or four significant digits was considered as the measured data. The accuracy of the sensor used in the experiment corresponded to the approximate number to four significant digits. Some examples used for the identification are shown in Table 3.

The force identification was carried out under the same conditions as those described in the previous section when one external force acted on the beam. When the data of two or three significant digits was used, the force could not be identified. When the data of three significant digits was used, there were 938 combinations where one external force acted on Node 11 and no forces acted on the other locations. The amount was less than $1026\left(={ }_{20} C_{3} \times 0.9\right)$, so we could not determine the final identification result. Looking at other combinations, we saw that there were 30 combinations where two external forces acted on Nodes 9 and 10 and no forces acted on the other locations, and 30 combinations where two external forces acted on Nodes 12 and 13 and no forces acted on the other locations. The amount was less than $154\left(={ }_{19} C_{2} \times 0.9\right)$, so we could not determine the final identification result. When the data of four significant digits was used, there were 1127 combinations, and we could determine the final identification result, that is, one external force of $10.00 \mathrm{~N}$ acted on Node 11 .

Next, when two external forces acted on the beam, the external force was identified under the same conditions as those described in the previous section. As in the case of one external force, when the data of two or three significant digits was used, the force could not be identified, but when the data of four significant digits was used, the correct result could be obtained. There were 171 combinations that satisfied the condition of two external forces, and as a result, we determined that one external force of $10.00 \mathrm{~N}$ acted on Node 9 and one of $12.00 \mathrm{~N}$ acted on Node 14 .

Here, when the data of three significant digits was used, there were 55 combinations where two external forces acted on Nodes 9 and 14 and no forces acted on the other

Table 3 Displacements at sensor locations with noise in the case of one excitation

\begin{tabular}{|c|c|c|c|c|}
\hline Approximate number & $x_{4}(\mathrm{~m})$ & $x_{8}(\mathrm{~m})$ & $x_{14}(\mathrm{~m})$ & $x_{18}(\mathrm{~m})$ \\
\hline Two significant digits & $4.0 \times 10^{-4}$ & $6.8 \times 10^{-4}$ & $6.8 \times 10^{-4}$ & $4.1 \times 10^{-4}$ \\
\hline Three significant digits & $4.00 \times 10^{-4}$ & $6.77 \times 10^{-4}$ & $6.80 \times 10^{-4}$ & $4.07 \times 10^{-4}$ \\
\hline Four significant digits & $4.000 \times 10^{-4}$ & $6.774 \times 10^{-4}$ & $6.803 \times 10^{-4}$ & $4.069 \times 10^{-4}$ \\
\hline
\end{tabular}


locations. The amount was less than $154\left(={ }_{19} C_{2} \times 0.9\right)$, so we could not determine the final identification result.

Finally, the force identification was carried out under the same conditions as those described in the previous section when three external forces acted on the beam. As in the previous cases, when the data of two or three significant digits was used, the force could not be identified, but when the data of four significant digits was used, the correct result could be obtained. There were 17 combinations that satisfied the condition of three external forces, and as a result, we determined that the external forces of $10.99 \mathrm{~N}$ at Node $9,12.00 \mathrm{~N}$ at Node 11 and $10.00 \mathrm{~N}$ at Node 15 acted.

Here, when the data of three significant digits was used, there were 50 combinations where two external forces acted on Nodes 10 and 15 and no forces acted on the other locations. The amount was less than $154\left({ }_{19} C_{2} \times 0.9\right)$, so we could not determine the final identification result.

As mentioned above, the correct identification results could be obtained by using the data of four significant digits in this study. If the data of three significant digits was used, in the case of one or two external forces, the correct results could be obtained by adjusting the criteria of determination of the final result. If the correct external force was unknown, the adjustment of the criteria was difficult. Moreover, in the case of three external forces, the correct result could not be obtained because the pattern with the best combinations was the one with two external forces.

\section{Experiment}

\subsection{Experimental setup}

The outline of the experimental setup is shown in Fig. 4. The exciter (IMV, VS-20-03) was connected with the beam through a load cell (PCB, 208B). The displacements were measured by displacement sensors (KEYENCE, EX-205), and the signal processing was carried out using specialized equipment (ONO SOKKI, Data Station).

The specifications were as follows:

Length: $1.0 \mathrm{~m}$, Moment of inertia: $1.80 \times 10^{-9} \mathrm{~m}^{4}$,

Young's modulus: 206GPa, Density: $7.65 \times 10^{3} \mathrm{~kg} / \mathrm{m}^{3}$,

Spring constant at Node 2: $27.4 \mathrm{kN} / \mathrm{m}$, Spring constant at Node 20: $26.4 \mathrm{kN} / \mathrm{m}$.

The masses of the connecting part between the beam and the spring were as follows:

Connecting element at Node 2: $0.371 \mathrm{~kg}$, Connecting element at Node 20: $0.367 \mathrm{~kg}$ and they were added into the mass matrix of the beam.

\subsection{Results and discussion}

The exciter was located at the center of the beam. The frequency and the magnitude of the external force were $7.0 \mathrm{~Hz}$ and $10.0 \mathrm{~N}$, respectively. The displacements by the mathematical model and the experiment are shown in Fig. 5. The solid line shows the analytical data, and the symbol (O) is the experimental data. As this figure shows, the mathematical model is sufficient to use for force identification.

The location and magnitude of the external force were identified using the measured data. Under the same criteria of the estimation of zero value and the estimation of the same value as the numerical example, that is, $3 \%$, we performed force identification. There were many

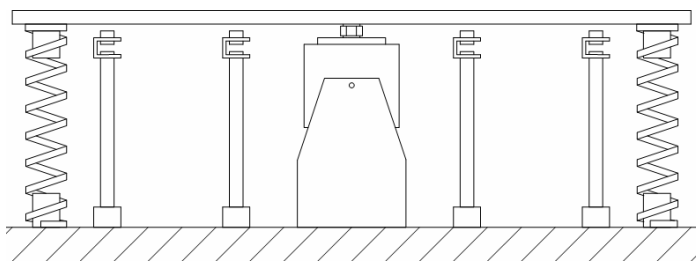

Fig.4 Outline of experimental setup 


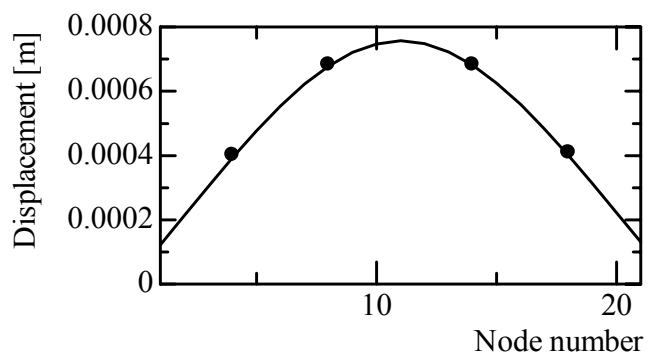

Fig.5 Displacement by analytical and experimental results

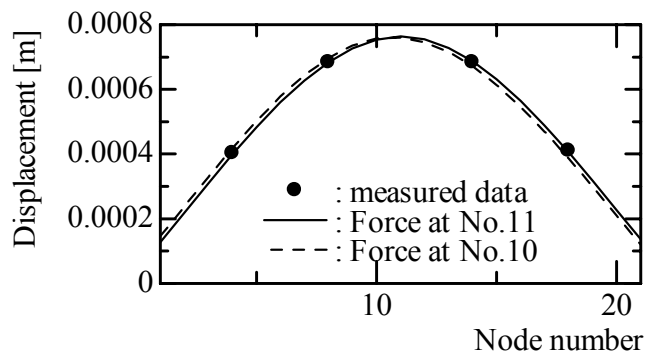

Fig.6 Displacement by identified and experimental results

patterns because of the measurement error and the modeling error, so identification could not be performed.

Next, we modified the criteria of the estimation to $10 \%$. As a result, the best possible case was one external force at Node 11 of 49 combinations, while the 2nd and 3rd possible cases were two external forces at Node 10 and Node 15 of 46 and 40 combinations, respectively. The difference of the 2 nd and the 3rd possibility was the magnitude of the external force. Our results showed that the patterns of the 1 st, the 2 nd and the 3 rd possibilities were quite different, so that the final result could not be achieved.

Next, we attempted a stepwise identification. At the first step, the location of the external force was roughly identified under the criteria of estimations of $30 \%$. Then, the magnitude of the external force was identified at the identified locations.

When the criteria was modified to $30 \%$, the best possible case was one external force at Node 11 of 242 combinations, and the 3rd possibility was the same result of 197 combinations. The difference was the magnitude of the external force. The 2 nd possibility was one external force at Node 10 of 241 combinations. From these results, we concluded that the external force acted at Node 11 or Node 10, and the magnitude of the external force was identified at those nodes. The magnitude of the external force was $9.99 \mathrm{~N}$ at Node 11 or $10.88 \mathrm{~N}$ at Node 10. The displacements are shown in Fig. 6. The error norm was defined as follows:

$$
R=\sqrt{\sum_{j=1}^{4}\left\{\left(x_{m}\right)_{j}-\left(x_{i d}\right)_{j}\right\}^{2}},
$$

where $\left(x_{m}\right)_{j}$ and $\left(x_{i d}\right)_{j}$ were the displacements of the $j$-th point of the measured data and the identified one, respectively. The norm at Node 11 was $7.0 \times 10^{-6} \mathrm{~m}$, while the one at Node 10 was $3.4 \times 10^{-5} \mathrm{~m}$. We concluded that one external force whose magnitude was $9.99 \mathrm{~N}$ acted at Node 11 .

As shown above, in the case of actual application of the proposed method, the stepwise approach is feasible when the locations of the external forces are identified under the easy criteria, and in these cases the magnitudes of the external forces are identified. 


\section{Conclusions}

We proposed a force identification method that was applicable to the case of unknown force locations. The number of external force was set according to the number of sensors. Then, the magnitudes of the external forces were identified for all combinations of force location. Finally, the locations of the external forces were determined by checking the number of combinations for every location pattern.

In the numerical example, the validity and the applicability of the proposed method were checked using the data without and with measurement error. In the experiment, we showed that the stepwise identification approach was feasible in actual applications.

In the numerical example and the experiment of this study, we carried out external force identification at a certain frequency that was lower than the first natural frequency. We confirmed that the correct results could be obtained at other frequencies lower than the first natural frequency in the numerical example. When the structure is excited at the high frequency and the higher-order vibration modes are excited, the property of the FRF matrix may be very much affected by the dominant vibration modes, the location of the acting force and the number and location of the sensors. In the actual application of the proposed method, the relationship of the natural frequency of the structure and the excitation frequency must be considered.

\section{References}

(1) The Japan Society of Mechanical Engineers ed., Computer Analysis of Inverse Problems, (1991), CORONA CO.,LTD.

(2) Kubo, S., Inverse Problems, (1992), BAIFUKAN CO.,LTD.

(3) Karl K.Stevens, Force Identification Problems an Overview, Proceedings of the 1987 SEM Spring Conference on Experimental Mechanics, Houston, TX, USA, (1987), pp.838-844.

(4) Hori, Y., Takasu, M., Honda, S., Sasaki, M. and Fujisawa, F., Estimation of location of exciting force inside the power equipment using an inverse problem of vibration, Proceedings of JSME Dynamics and Design Conference 2000, (2000). Paper No.445 on CD-ROM.

(5) A.S.Sekhar, Crack identification in a rotor systema model-based approach, Journal of Sound and Vibration, 270, (2004), pp.887-902.

(6) Kawamura, S., Hayakawa, Y, Hossain Md. Zahid, Minamoto, H., Development of force identification method based on equation of motion, Proceedings of JSME Dynamics and Design Conference 2006, (2006), Paper No.643 on CD-ROM.

(7) Hori, Y., Suzuki, R., Kawase, S., Sasaki, M. and Fujisawa, F., Estimation of location of exciting force inside the power equipment using an inverse problem of vibration, Proceedings of JSME Dynamics and Design Conference 2001, (2001), Paper No.214 on CD-ROM. 